\title{
Análisis Biométrico de las Arterias Comunicante Anterior y Cerebral Anterior en el Segmento Precomunicante del Círculo Arterial Cerebral
}

\author{
Biometrical Analysis of the Anterior Communicating Artery and the Anterior Cerebral Artery in \\ the Precommunicating Segment of the Cerebral Arterial Circle \\ "Eduardo Mandiola; "Eduardo Alarcón; "*Mariano del Sol; **Enrique Olave; "C. Montero; "P. Sanhueza \& "Juan Carlos Oñate
}

MANDIOLA, E.; AlARCón, E.; DEL SOL, M.; OLAVE, E.; MONTERO, C.; SANHUEZA, P. \& OÑATE, J. C. Análisis biométrico de las arterias comunicante anterior y cerebral anterior en el segmento precomunicante del círculo arterial cerebral. Int. J. Morphol., 23(2):171$176,2005$.

RESUMEN: La conformación del círculo arterial cerebral tiene relevancia en la clínica neuroquirúrgica por la relación compleja que presentan las arterias que lo originan y su gran variabilidad. Debido a la alta frecuencia con que se observan aneurismas en las arteria comunicante anterior y en el segmento precomunicante (A1) de la arteria cerebral anterior se efectuó un análisis biométrico de ellas.

El trabajo se realizó en 36 cerebros frescos procedentes de especímenes cuyos datos bioantropológicos estaban registrados. El calibre de la arteria comunicante anterior fue de $1.68 \mathrm{~mm}$ y la longitud, en promedio, de $2.50 \mathrm{~mm}$. En el lado derecho el segmento A1 de la arteria cerebral anterior tenía una longitud de $12.91 \mathrm{~mm}$ y un calibre de $2.39 \mathrm{~mm}$. En el lado izquierdo, la longitud de este segmento fue de $12.77 \mathrm{~mm}$ y presentó un calibre de $2.46 \mathrm{~mm}$. En un $29 \%$ de las muestras analizadas, se presentaron arterias comunicantes dobles, con una longitud de $2.92 \mathrm{~mm}$ y un calibre de $0.95 \mathrm{~mm}$.

Se observó variabilidad en los componentes que constituyen el círculo arterial cerebral, cuando se consideran variables como lado, sexo e índice cefálico.

PALABRAS CLAVE: Arteria comunicante anterior; Arteria cerebral anterior; Círculo arterial del cerebro; Índice cefálico.

\section{INTRODUCCIÓN}

La conformación vascular del círculo arterial cerebral (Polígono de Willis), ha sido estudiada por diversos autores clásicos y por anatomistas clínicos, debido a su relevancia en intervenciones quirúrgicas

Todo este conjunto vascular puede involucrar alteraciones funcionales cuando su conformación no es adecuada, ya sea por su desarrollo o por otros factores que lo afecten, como es el caso de procesos expansivos, ya que nutren estructuras corticales y centrales de los hemisferios cerebrales.

Además, se ha observado con cierta frecuencia que hay numerosas variaciones en el calibre y trayecto de las arterias componentes del círculo (Duroux et al., 1953; González, 1959; Barbosa et al., 1969; Sengupta, 1975; Lazorthes et al., 1979; Kamath, 1980; Orlandini et al., 1985; Milisavljevic et al., 1986; Overbeeke et al., 1991).

No es nuevo el concepto que las arterias contiguas a los troncos de los nervios craneales son una causa de los síntomas de diversas disfunciones de éstos (Janetta, 1967; Jiménez-Castellanos et al., 1992; Cavdar et al., 1995 ).

Los instrumentos de magnificación quirúrgica para operaciones en esta área, ha creado la necesidad de un mejor conocimiento de la anatomía microquirúrgica.

\footnotetext{
* Universidad Andrés Bello. Dpto. Ciencias Biológicas. Santiago, Chile.

* Universidad de La Frontera, Temuco, Chile.

Parcialmente financiado por Proyecto DI-UNAB-03-03. Universidad Andrés Bello. Chile.
} 
Mandiola et al., 19941999, 2002 y 2004) estudiaron detalles anatómicos de las arterias del sistema vértebro -basilar, tomando en consideración el índice cefálico-horizontal (IC-H), encontrando variabilidad importante al comparar los diversos grupos.

Nuestra investigación es una contribución para la identificación de las muchas características y variaciones que presentan las arterias componentes del círculo arterial cerebral, considerando lado, sexo y el índice cefálico (IC-H), factor que introducimos, que creemos importante y que no ha sido considerado por otros autores, es que a través de los cuales, se investiga la variabilidad arterial en la arteria comunicante anterior (AcoA) y el segmento precomunicante de la arteria cerebral anterior (ACA, A1).

\section{MATERIAL Y MÉTODO}

Estudiamos 36 encéfalos seleccionados de cadáveres frescos de individuos, obtenidos de autopsia, de ambos sexos, de los cuales 25 correspondían al sexo masculino y 11 al femenino, con edades que fluctuaban entre 18 y 94 años, sin evidencias de enfermedad vascular del sistema nervioso ni de procesos expansivos.

Se estudiaron los encéfalos, desde el punto de vista biométrico, correlacionando los datos obtenidos de dimensiones cefálicas de los especímenes, con longitud y calibre de las arterias que conforman el círculo arterial cerebral, usando caliper digital (Mitutoyo), y utilizando lupa de $4 \mathrm{X}$ con autoiluminación.
El segmento proximal de la arteria cerebral posterior (ACP), precomunicante se denominó P1, asimismo el segmento proximal de la ACA ( precomunicante ) se denominó A1. Los casos se diseñaron y fotografiaron con cámara digital Sony P-50.

Se realizaron las mediciones cefálicas longitud A-P máxima y ancho máximo (biparietal), para el cálculo del índice cefálico horizontal (IC-H) según Martín-Saller (1957). Las características de la muestra, permitieron dividirla en dos grupos: braquicéfalos $(\mathrm{BH})$ desde 81.0 hasta más de 85.5 y dólico-mesocéfalos (DM) hasta 80.9 de acuerdo al índice.

Todos los valores obtenidos fueron analizados estadísticamente, a través del cálculo de las medias con su desviación estándar y por correlación de Pearson usando el software Prism V.4.0, de todos los parámetros que se midieron: calibre, longitud de cada arteria componente del círculo, diámetros cefálicos y cálculo del índice cefálico (IC). Las diferencias significativas fueron evaluadas a través del test de Student para las medias.

\section{RESULTADOS}

Tabla I. Valor medio y desviación estándar ( $\mathrm{mm}$ ) del calibre y longitud del segmento anterior del circulo arterial del cerebro, según lado.

\begin{tabular}{llccc} 
Arteria & & $\begin{array}{c}\text { Lado derecho } \\
(\mathrm{mm})\end{array}$ & $\begin{array}{c}\text { Lado izquierdo } \\
(\mathrm{mm})\end{array}$ & $\mathrm{p}$ \\
\hline \multirow{2}{*}{ ACA $(\mathrm{A} 1)$} & Cal ibre & $2.37 \pm 0.68$ & $2.42 \pm 0.75$ & 0.7679 \\
\multirow{2}{*}{ ACoA } & Longitud & $12.86 \pm 1.58$ & $12.62 \pm 1.96$ & 0.5692 \\
& Calibre & \multicolumn{2}{c}{$1.68 \pm 0.81$} & \\
& Longitud & \multicolumn{2}{c}{$2.50 \pm 1.52$} \\
\hline
\end{tabular}

Tabla II. Valor medio y desviación estándar ( $\mathrm{mm})$ del calibre y longitud del segmento anterior del circulo arterial del cerebro, según sexo.

\begin{tabular}{llccc}
$\begin{array}{l}\text { Nombre } \\
\text { Arteria }\end{array}$ & Calibre & $\begin{array}{c}\text { Hombre } \\
(\mathrm{mm})\end{array}$ & $\begin{array}{c}\text { Mujer } \\
(\mathrm{mm})\end{array}$ & $\mathrm{p}$ \\
\hline ACA & $2.37 \pm 0.72$ & $2.45 \pm 0.70$ & 0.6753 \\
& Longitud & $12.81 \pm 1.86$ & $12.57 \pm 1.58$ & 0.6149 \\
ACoA & Calibre & $1.53 \pm 0.68$ & $1.05 \pm 0.81$ & $0.0147 * *$ \\
& Longitud & $2.81 \pm 1.76$ & $2.33 \pm 1.32$ & 0.2770 \\
\hline
\end{tabular}

$(* *=$ diferencia significativa $)$

Tabla III. Valor medio y desviación estándar ( $\mathrm{mm}$ ) del calibre y longitud del segmento anterior del circulo arterial del cerebro, según índice cefálico.

\begin{tabular}{llccc}
$\begin{array}{l}\text { Nombre } \\
\text { Arteria }\end{array}$ & & \multicolumn{1}{c}{$\begin{array}{c}\mathrm{BH} \\
(\mathrm{mm})\end{array}$} & $\begin{array}{c}\mathrm{DM} \\
(\mathrm{mm})\end{array}$ & $\mathrm{p}$ \\
\hline ACA & Calibre & $2.34 \pm 054$ & $2.43 \pm 0.79$ & 0.6205 \\
& Longitud & $13.03 \pm 2.11$ & $12.58 \pm 1.57$ & $0.3219^{* *}$ \\
& & & & \\
ACoA & Calibre & $1.38 \pm 0.69$ & $1.37 \pm 0.80$ & 0.5853 \\
& Longitud & $2.52 \pm 1.73$ & $2.75 \pm 1.61$ & 0.9590 \\
\hline
\end{tabular}

$\left({ }^{* *}=\right.$ diferencia significativa $)$ 
Tabla IV. Valor medio y desviación stándar ( $\mathrm{mm})$ del calibre y longitud del segmento anterior del círculo arterial del cerebro, según modalidad de AcoA.

\begin{tabular}{llccc}
$\begin{array}{l}\text { Nombre } \\
\text { Arteria }\end{array}$ & \multicolumn{1}{c}{$\begin{array}{c}\text { DOBLE } \\
(\mathrm{mm})\end{array}$} & $\begin{array}{c}\text { SINGLE } \\
(\mathrm{mm})\end{array}$ & $\mathrm{p}$ \\
\hline ACoA & Calibre & $0.95 \pm 0.38$ & $1.68 \pm 0.81$ & $0.0010^{* *}$ \\
& Longitud & $2.92 \pm 1.73$ & $2.49 \pm 1.55$ & 0.3975 \\
& & & & \\
ACA & Calibre & $2.56 \pm 0.55$ & $2.33 \pm 0.76$ & 0.2440 \\
& Longitud & $11.94 \pm 1.49$ & $13.02 \pm 1.79$ & $0.0254 * *$ \\
\hline
\end{tabular}

Al comparar los resultados obtenidos en los lados derecho e izquierdo de la ACA (A1), de su calibre y longitud, no se observan diferencias estadísticamente significativas. La ACoA presenta una dispersión bastante alta en sus valores (Tabla I).

En cambio, se apreció una diferencia significativa ( $\mathrm{p}=0.0147)$ en el calibre de la ACoA con relación al sexo. En el sexo masculino, es posible observar un promedio más alto en esta dimensión. (Tabla II).

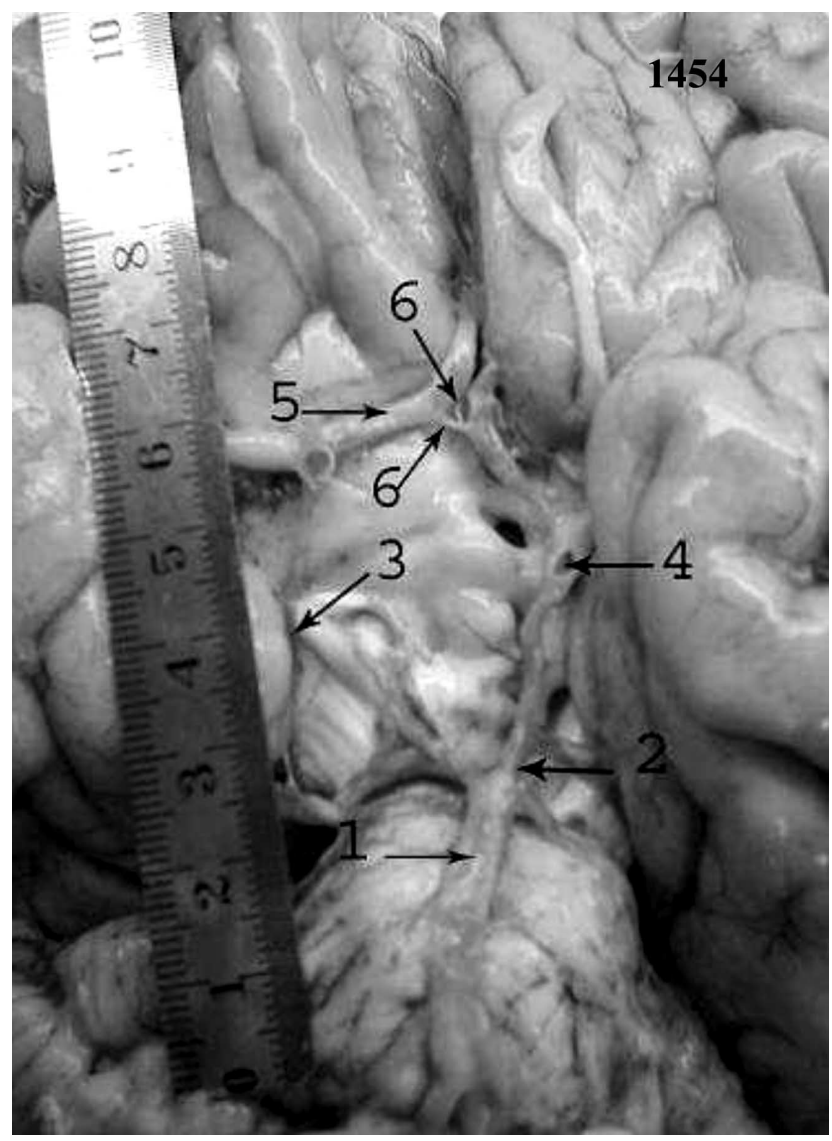

Fig. 1. Fotografía de la superficie ventral del círculo arterial de la base del cerebro. Se observa arteria comunicante anterior doble, siendo la más anterior hipoplástica. 1. A. basilar; 2. A. cerebral posterior; 3. A. comunicante posterior; 4 . A. carótida interna; 5 . A. cerebral anterior; 6. Aa. comunicantes anteriores.
En lo que respecta al IC, no se observaron diferencias significativas con relación al calibre y longitud de la ACA (A1) y ACoA entre los BH y DM. (Tabla III).

Es destacable en nuestra muestra la presencia de 10 círculos arteriales del cerebro con presencia de ACoA dobles $(29 \%)$, en las cuales, a su vez, se registraron 4 casos

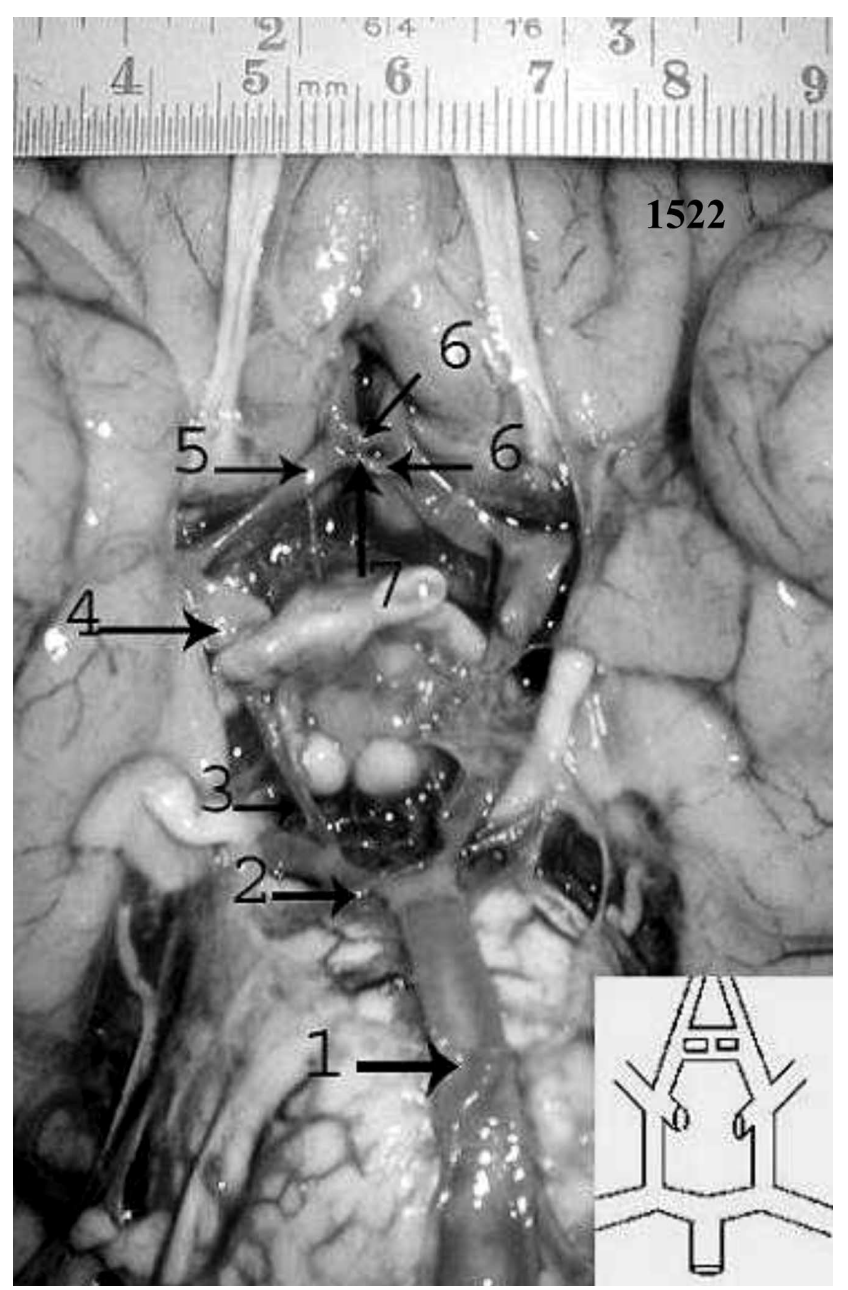

Fig. 2. Fotografía y diagrama de la superficie ventral del círculo arterial de la base del cerebro. Se observa una arteria comunicante anterior doble y un puente anastomótico. 1. A. basilar; 2. A. cerebral posterior; 3. A. comunicante posterior; 4. A. carótida interna; 5. A. cerebral anterior; 6. Aa. comunicantes anteriores; 7. Puente anastomótico. 
(12\%) con ambas ACoA hipoplásticas (menos de 1mm de calibre), y 5 casos (15\%) con una de las ACoA hipoplásticas. (Fig.1).

En las ACoA observamos arterias hipoplásticas en 14 $\operatorname{casos}(41 \%)$.

Además, se obtuvieron diferencias en el calibre de la ACoA, modalidad doble en relación a la simple ( $\mathrm{p}=0.001)$, y en la longitud de la ACA en aquellas que poseían ACoA doble en relación a las simples $(\mathrm{p}=0.0254)$. (Tabla IV).

Se observó 1 caso (3\%), de arteria hipoplástica unilateral y 1 caso (3\%), de arterias hipoplásticas bilaterales en la ACA.

En 1 de nuestros casos (3\%), se presentó un puente anastomótico entre dos ACoA.(Fig.2).

Una observación importante corresponde a 1 caso (3\%), en el cual se visualizó una arteria intermedia a las ACAs, que se originaba a partir de la ACoA y, posteriormente, ésta se dividía en dos y reemplazaba a las ACAs, transformándose en arteria pericallosa, y las ACAs originales seguían el recorrido de una arteria callosa marginal.

\section{DISCUSIÓN}

La incidencia de aneurismas en la ACoA (30\%) o en la ACA (A1) reportados por Karazincir et al. entre otros, se debería a variaciones en el círculo arterial cerebral según Fujimoto \& Tanaka (1989) ya sea por hipoplasia o por agenesia de éstas.

La presencia ACoA dobles (29\% en nuestra serie) aumentaría la probabilidad de aneurismas. Así, Aydin et al. (1997) reportaron que el 60\% de los pacientes operados presentaban variaciones anatómicas en la vecindad de la ACoA, dentro de las cuales hay un $8.3 \%$ de ACoAs dobles en pacientes con aneurismas.
Embriológicamente, la ACoA proviene de una formación secundaria de puentes anastomóticos entre las ACAs (Testut \& Latarjet, 1964) lo que explicaría la presencia de otra anastomosis dando origen a esta segunda ACoA, motivo por el cual estas nuevas formaciones de ACoAs tienen menor calibre (0.95 $\pm 0.38 \mathrm{~mm})$ en relación a la ACoA común $(1.68 \pm 0.81 \mathrm{~mm})$.

En nuestro estudio las ACoAs dobles se encontraron en 4 mujeres y en 6 hombres, no teniendo relación el sexo, ni tampoco el índice cefálico, ya que el $50 \%$ corresponde a DM y el $50 \%$ a $\mathrm{BH}$.

El rango del calibre de la ACA estuvo entre 0.77-3.54 mm y el de la longitud 9.57-17.33 mm; el calibre de la ACoA fue entre 0.68-3.11mm y la longitud 0.8-5.41 mm. El 90\% de la ACoA dobles tienen un diámetro menor a $1 \mathrm{~mm}$ (hipoplásticas) y, del total de las ACoAs de los distintos circulos, el 36\% sería hipoplástico, lo que se confirma con los estudios de Krupa et al. (1998) qquienes señalan un $30 \%$ con esta característica.

En relación a la presencia de ACoAs dobles, se observaron 2 disposiciones de éstas; en una ambas ACoAs se encontraban en forma paralela (4 casos) y en la otra convergían hacia una ACA (6 casos ).

La presencia de ACA hipoplásticas en pacientes con aneurismas, es de $26.6 \%$ en el estudio de Karazincir et al., y en nuestro trabajo fue sólo de un $6 \%$.

Al igual que Karazincir et al., Aydin et al. y Kakou et al. (2000) han descrito la aparición de una arteria ácigos pericallosa y una arteria mediana del cuerpo calloso, encontramos un círculo en el cual se observaban estas dos características.

Estos resultados son un aporte al conocimiento de nuestra etnicidad y consideración en la clínica através de parámetros, tales como: lado del círculo, sexo e índice cefálico, en los cuales se observa gran variabilidad entre las arterias que componen el círculo arterial cerebral.

MANDIOLA, E.; ALARCÓN, E.; DEL SOL, M.; OLAVE, E.; MONTERO, C.; SANHUEZA, P. \& OÑATE, J. C. Biometyrical analise of the anterior communicating artery and the anterior cerebral artery in the precommunicating segment of the cerebral arterial circle. Int. J. Morphol., 23(2):171-176, 2005.

SUMMARY: The conformation of the cerebral arterial circle has relevance in the clinical neurosurgery for the complex relate that present the arteries that originate it and its great variability. Due to the high frequency with that aneurysms are observed in the anterior communicant artery and the anterior cerebral artery in the precommunicating segment (A1), we have decided to make an analysis biometrical in them.

The work had done in 36 available fresh brains in the laboratories and they come from specimens whose data bioanthropological were registered. The caliber of the anterior communicating artery corresponds to $1.68 \mathrm{~mm}$ and the longitude to $2.50 \mathrm{~mm}$. The segment A1 of the anterior cerebral artery it corresponds to a longitude of $12.91 \mathrm{~mm}$ and it presents a caliber of $2.39 \mathrm{~mm}$ in the right side. In the left side the longitude of this segment is of $12.77 \mathrm{~mm}$ and it presents a caliber of 2.46 . In $29 \%$ of the analyzed samples, they register double communicating arteries, with a longitude of $2.92 \mathrm{~mm}$ and a caliber of $0.95 \mathrm{~mm}$. Variability of the components is observed that they constitute the cerebral arterial circle when is considered: side, sex and cephalic index

KEY WORDS: Anterior communicating artery; Anterior cerebral artery; Cerebral arterial circle; Cephalix index. 


\section{REFERENCIAS BIBLIOGRÁFICAS}

Aydin, I. H.; Takci, E.; Kadioglu, H. H.; Tuzun, Y.; Kayaoglu, C. R. \& Barlas, E. Vascular variations associated with anterior communicating artery aneurysms-an intraoperative study. Minim. Invasive neurosurg., 40(1):17-21, 1997.

Barbosa, L.; Purriel, J. A.; Meerhoff, W. \& Medoc, J. El polígono de Willis y sus variaciones. Acta Neurol. Latinoamer., 15:224-36, 1969.

Cavdar, S.; Dalcik, H.; Yalin, A. A rare case of the posterior inferior cerebellar artery. Acta Anat (Basel)., 152(3):234-6, 1995.

Duroux, M. M. P. E.; Dujol, H.; Avet, J.; Gabrielle, C. L. A propos du polygone de Willis. C. R. Ass, Anat., 79:328-38, 1953

Fujimoto, K. \& Tanaka, O. Morphological examination of the circulus arteriosus cerebri human (circle of Willis). Anterior and posterior communicating arteries. Anat. Anz., 168(2):145-54, 1989.

González, A. A. Circulo arterial de Willis. Sus medidas externas y variaciones anatómicas. Acta Neurol. Latinoamer., 5: 1-6, 1959.

Janetta, P. J. Arterial compression of the trigeminal nerve at the pons in patients with trigeminal neuralgia. $J$. Neurosurg., 26:159-62, 1967.

Jimenez-Castellanos, Jr; Armona; Talina, J.; JimenezCastellanos, J. Sr. Gross (mesoscopic) and applied anatomy of the anterior inferior cerebellar artery in man with special reference to its course through the cerebellopontine angle region. Acta Anat., 143(3):1827, 1992

Kakou, M.; Destrieux, C. \& Velut, S. Microanatomy of pericallosal artery complex. J. Neurosurg., 93:667-75, 2000

Kamath, S. Observations en the length and diameter of vessels forming the circle of Willis. J. Anat., 133(3): 419-23, 1981.

Karasincir, S.; Ada, E.; Sarilmaz, A.; Yalcin, O.; Vidinli, B. \& Sabin, E. Frequency of vascular variations and anomalies ccompanying intracranial aneurysms. Tani Girisim Radyol., 10(2):103-9, 2004.
Krupa, B. \& Krupa, U. Variations of the anterior communicating artery. Folia Morphol., 57(3):233-40, 1998.

Lazorthes, G.: Gouaze, A.; Santini, J. J. \& Salamon, G. Le cercle arteriel du cerveau (circulus arteriosus cerebri). Anat. Clinica, 1:241-257, 1979.

Mandiola, E.; del Sol, M.; Sanz, M. E.; Olave, E.; Gabrielli, C. \& Prates, J. C. Distribución Arterial en la Fisura Horizontal del Cerebelo. Rev. Chil. Anat., 14(2):177-81,1996.

Mandiola, E.; del Sol, M.; Henriquez, J.; Prates, J. C. Observaciones Anatómicas de la Parte Ambiens de la Arteria Cerebelar Superior. Rev. Chil. Anat., 13(1):616, 1995.

Mandiola, E.; del Sol, M., Sanz, M. E.; Olave, E.; Gabrielli, C. \& Prates, J. C. Características Anatómicas de las Ramas Hemisféricas de la Arteria Cerebelar Superior. Rev. Chil. Cs. Méd. Biol., 6(2):75-80, 1996.

Mandiola, E.; del Sol, M.; Olave, E.; Gabrielli, C.; Mizusaki, C. \& Prates, J.C. Bifurcation of the Basilar Artery and its Relationship with the Superior Cerebellar Artery at the Proximal Portion of the Anterior Pontine Segment. Braz. J. Morphol. Sci., 15 (1):29- 33, 1998 .

Mandiola, E.; del Sol, M.; Olave, E.; Gabrielli, C. \& Prates, C. Consideraciones Anatómicas de la Arteria Basilar. Rev. Chil. Anat.,13(2):189-94, 1995.

Mandiola, E.; del Sol, M.; Olave, E.; Gabrielli, C. \& Prates, J. C. Consideraciones anatómicas de la arteria basilar. Rev. Chil. Anato., 13(2):189-94, 1995

Mandiola, E.; del Sol, M.; Sanz, M.E.; Olave, E.; Gabrielli, C. \& Prates, J.C. Variabilidad Anatómica de las Ramas Medial y Lateral de la Arteria Cerebelar Superior. Rev. Chil. Anat., 15 (1):85-91, 1997.

Mandiola, E.; del Sol, M.; Sanz, M. E.; Olave, E.; Gabrielli, C. \& Prates, J.C. Distancia desde el origen de la Arteria Cerebelar Superior hasta la bifurcación de la Arteria Basilar y Origen de la Arteria Cerebelar Anterior Inferior. Rev. Chil. Anat., 14(1):67-72, 1996.

Mandiola, E.; Sanz, M. E.; Gabrielli, C. \& Prates, J. C. 
Diámetros de las Arterias Cerebelar Superior y Cerebelar Anterior-Inferior en el Segmento Pontino-Anterior. Rev. Chil. Anat., 16 (1):33-8, 1998.

Mandiola, E.; Sanz, M.E.; Reumay, P.; del Sol, M.; Olave, E. \& Prates, J.C. Biometría de la arteria cerebelar anterior-inferior en el segmento pontino-anterior y lateral. Rev. Chil. Anat., 17 (2):161-7, 1999.

Mandiola, E.; del Sol, M. \& Prates, J. C. Relación Anatómica de la Arteria Cerebelar Superior con los Nervios Oculomotor y Troclear considerando el Grupo Etnico e Indice Cefálico. Rev. Chil. Cs. Med. Biol., 5(1):5-10, 1995.

Mandiola, E. \& Prates, J. C. Características Anatómicas de la Arteria Cerebelar Superior en el Hombre. Parte Pontina Anterior. Rev. Chil. Anat., 12(1):25-32, 1994.

Mandiola, E.; del Sol, M.; Olave, E. \& Prates, J. C. Relaciones Anatómicas de la Arteria Cerebelar Superior con el Nervio Trigémino. Rev. Chil. Anat., 12(4):141-6, 1994.

Martin, R. \& Saller, K. Lehrbuch der Anthropologie. Stuttgart, Gustav Fischer Velgar, 1957. V.1. pp. 317-79.

Milisavljevic, M.; Marinkovic, S.; Lolic-Draganic, V. \& Djordjevic, L. Anastomoses in the Territory of the Posterior Cerebral Arteries. Acta Anat., 127:122-5, 1986.

Orlandini, G.; Ruggiero, C.; Orlandini, S. Z. \& Gulisano, M. Blood vessel size of circulus arteriosus cerebri (circle of Willis): A Statistical Research on 100 Human Subjects. Acta Anat., 123:72-6, 1985.

Overbeeke, V. J. J.; Hillen, B. \& Tulleken, C. A. A Comparative study of the circle of Willis in fetal and adult life. The configuration of the posterior bifurcation of the posterior communicating artery. Bull. Assoc. Anat., 75(229)ss:123-6, 1991.

Sengupta, R. P. Anatomical variations in the origin of the posterior cerebral artery demostrated by carotid angiography and their significance in the direct surgical treatment of posterior communicating aneurysms. Neurochirurgia, 18(2):33-42, 1975.

Testut, L. \& Latarjet, A. Tratado de anatomía humana. Barcelona, Salvat, 1964. V. 2.
Dirección para correspondencia : Prof. Dr. Eduardo Mandiola

Facultad de Ciencias de la Salud Depto. de Ciencias Biologicas Universidad Andrés Bello, República 217, Piso 2. Santiago, CHILE

E-mail : emandiola@unab.cl

Recibido : 08-01-2005

Aceptado : 15-03-2005 